\title{
Úlcera marginal perforada tras bypass gástrico. ¿Cuál es el manejo ideal? A propósito de un caso
}

\author{
Perforated marginal ulcer after gastric bypass. What is the ideal management? \\ About a case \\ Luisa F. Mogollón ${ }^{1}$, Andrés Mendoza-Zuchini, Luis F. Cabrera³, Mauricio Pedraza², \\ Gabriela Fuente-Navarrete 4 y Cristina Jiménez ${ }^{4}$ \\ ${ }^{1}$ Departamento de Cirugía General, Fundación Universitaria de Ciencias de la Salud; '2Departamento Cirugía General, Universidad El Bosque; \\ ${ }^{3}$ Departamento Cirugía General, Fundación Santafé de Bogotá; ${ }^{4}$ Departamento de Medicina, Universidad El Bosque. Bogotá, Colombia
}

\section{Resumen}

En la última década, las úlceras marginales en pacientes tras un bypass gástrico se han convertido en un problema mucho más frecuente, y hoy en día aún no está clara su etiología. Se han descrito numerosos factores de riesgo, tales como un alto consumo de tabaco o de antiinflamatorios no esteroideos (AINE), entre otros. Presentamos el caso de una paciente con úlcera gástrica perforada en el remanente gástrico, con antecedente de bypass gástrico hace 4 años, tabaquismo y uso de AINE, por lo que se lleva a manejo laparoscópico con parche de Graham, adecuada evolución clínica y resolución de la sintomatología.

Palabras clave: Bypass gástrico. Laparoscopia. Perforación. Úlcera péptica.

\begin{abstract}
In the last decade, marginal ulcers in post-gastric bypass patients have become a much more frequent problem. Multiple risk factors have been described, such as high consumption of tobacco or high consumption of nonsteroidal anti-inflammatory drugs (NSAIDs), among others. We present a case report of a patient with perforated gastric ulcer in the gastric pouch, with a history of gastric bypass 4 years ago associated with smoking and NSAIDs, so it is taken to laparoscopic management with Graham's patch management, adequate clinical evolution and resolution of symptoms.
\end{abstract}

Key words: Gastric bypass. Laparoscopy. Perforation. Peptic ulcer.

\section{Introducción}

El bypass gástrico es una de las diversas técnicas de cirugía bariátrica que hay en la actualidad, la cual busca reducir la morbimortalidad asociada a la obesidad mórbida'.
Este método quirúrgico, en personas que cursan con dislipidemias graves, diabetes mellitus tipo 20 son gravemente obesos, ha demostrado ser más eficaz y duradera que otras alternativas médicas y farmacológicas ${ }^{2}$.

La úlcera marginal es una de las complicaciones poco frecuentes que existen en el bypass gástrico con 
reconstrucción en $Y$ de Roux. Consiste en una ulceración cercana a la anastomosis gastroyeyunal del bypass gástrico y clásicamente en la mucosa yeyunal ${ }^{1}$. Puede aparecer desde el primer mes posoperatorio hasta incluso 6 años tras el procedimiento². Por ello, su tipo de presentación se clasifica según el tiempo de aparición, denominándose úlcera marginal temprana la frecuentemente vista en una endoscopia de control después del primer mes posoperatorio y úlcera marginal tardía a la evidenciada después del primer $a \tilde{n} 0^{3}$.

El 95\% de los pacientes que cursan con esta patología la desarrollaran en el primer año posoperatorio, y tan solo el $16 \%$ la presentará del primer al sexto mes. Para el tratamiento de la úlcera marginal perforada, la técnica más recomendada es el reforzamiento con parche omental mediante laparoscopia, ya que ha demostrado mayor efectividad y seguridad en caso de rotura de la úlcera con sangrado masivo ${ }^{4-7}$.

\section{Caso clínico}

Mujer de 38 años con antecedente de bypass gástrico realizado hace 4 años como tratamiento de obesidad mórbida con un índice de masa corporal previo de 42, que cursa con diagnóstico de síndrome de apnea obstructiva del sueño y discopatía; además, es consumidora crónica de antiinflamatorios no esteroideos (AINE) y 2 cajas diarias de cigarrillos, sin otros antecedentes relevantes.

Ingresa por un cuadro clínico de 12 horas de evolución, consistente en dolor epigástrico inicialmente de intensidad 9/10 sin sitio de irradiación, con posterior mejoría sintomática y con agudización en las últimas 2 horas de dolor generalizado asociado a múltiples episodios eméticos.

En la exploración física muestra una presión arterial de $110 / 70 \mathrm{mmHg}$, una frecuencia cardiaca de 110 latidos por minuto y una frecuencia respiratoria de 20 ${ }^{*}$ min. A la inspección el abdomen es globoso, no se auscultan soplos, y presenta dolor a la palpación profunda en el epigastrio sin signos de irritación peritoneal. Se le realiza radiografía de tórax en la que se evidencia neumoperitoneo en el espacio subdiafragmático derecho (Fig. 1).

Se indica revisión laparoscópica por sospecha de úlcera péptica perforada. Se realiza una exploración por cuadrantes de la cavidad abdominal, se lleva a cabo una prueba de azul de metileno por sonda nasogástrica y se identifica la úlcera marginal perforada en el pouch gástrico (Fig. 2). Se decide realizar

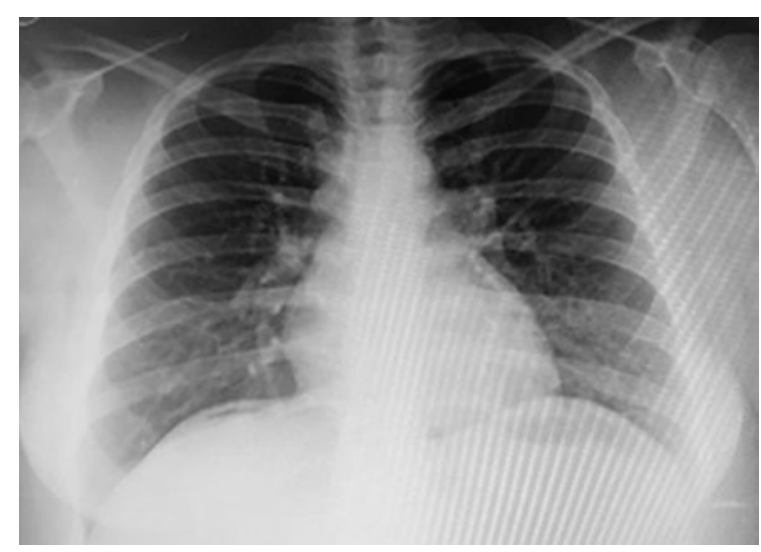

Figura 1. Radiografía de tórax en la cual se evidencia neumoperitoneo en el espacio subdiafragmático derecho.

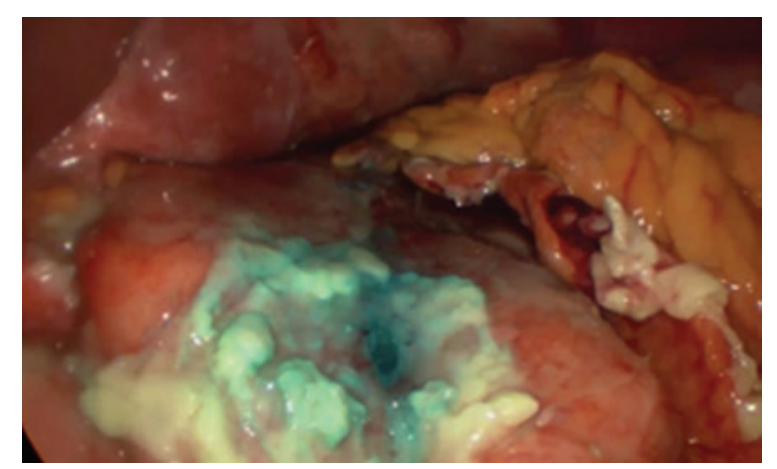

Figura 2. Úlcera marginal perforada con extravasación de azul de metileno.

biopsia de los bordes de la úlcera y parche de Graham por laparoscopia con fijación del colgajo de epiplón con polidoxanona 3-0 (Fig. 3).

La evolución posoperatoria fue adecuada y egresó al segundo día de hospitalización, sin complicaciones asociadas al procedimiento quirúrgico y con una adecuada tolerancia a la vía oral, por lo que se decide manejar de forma ambulatoria con un inhibidor de la bomba de protones y recomendaciones de cambios en el estilo de vida. El reporte de patología de las biopsias que se tomaron fue negativo para malignidad y se realizó seguimiento con resolución completa de la úlcera evidenciada en las endoscopias de control.

\section{Discusión}

La obesidad mórbida es uno de los mayores problemas en el mundo actualmente. Es por esto que la cirugía bariátrica se ha ido posicionando como el mejor 


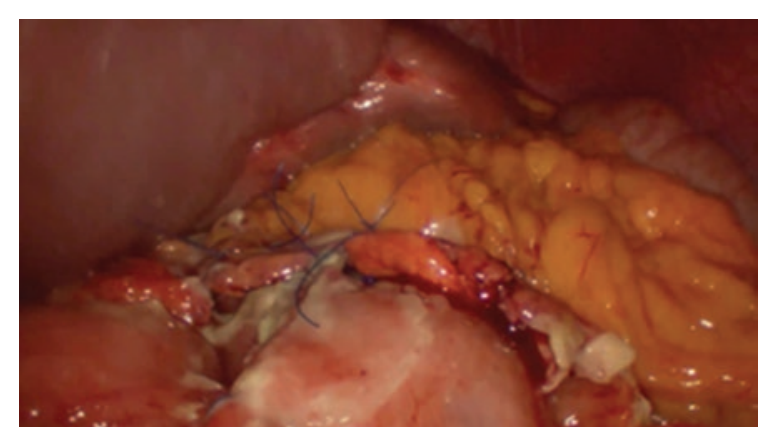

Figura 3. Parche de Graham por vía laparoscópica.

tratamiento para la obesidad mórbida y su comorbilidad. En estos pacientes, el bypass gástrico con reconstrucción en $Y$ de Roux puede ser más efectivo y puede mantener a más largo plazo la reducción de peso y evitar el reflujo gastroesofágico ${ }^{4-6,8}$.

La aparición de una úlcera marginal anastomótica es una complicación tardía del bypass gástrico, y aunque su etiología aún es desconocida, usualmente es más frecuente en el lado yeyunal de la anastomosis, hasta en un $16 \%$ en el caso de la $Y$ de Roux ${ }^{8,9}$ y un $14.6 \%$ en el caso del mini bypass gástrico ${ }^{10}$. La mayoría ocurren entre 1 y 6 meses después de la cirugía, y hasta el $95 \%$ de los pacientes que van a desarrollarla la presentarán en el primer año posoperatorio ${ }^{11}$.

La producción normal de ácido en el estómago es de 2-3 I/día y está estimulada por la acción parasimpática de la acetilcolina desde el nervio vago, la estimulación de la gastrina en las células $\mathrm{G}$ del antro 0 la estimulación de la histamina desde las células gástricas. Estos factores inducen la acción de la bomba $\mathrm{K}^{+} / \mathrm{H}^{+}$ATPasa sobre la superficie apical, secretando entonces hidrogeniones hacia la luz gástrica. Las células del epitelio gástrico tienen como función secretar moco y bicarbonato en respuesta a la irritación del epitelio de revestimiento o la estimulación colinérgica y de prostaglandinas. El moco actúa como barrera contra el ácido y el bicarbonato sirve como tampón de proximidad. Sin embargo, este equilibrio se puede afectar por varios factores, como el consumo crónico de AINE o la infección por Helicobacter pylori, dando como resultado la aparición de lesiones y posibles ulceraciones en el epitelio.

Por todo lo anterior, no es frecuente el desarrollo de úlceras en el pouch gástrico, ya que, al resecar el antro, no debería producirse la cascada mencionada anteriormente, y por esto no deberían desarrollarse úlceras a este nivel ${ }^{12}$.
Su incidencia es desconocida, pues su presentación en la mayoría de los casos es asintomática; sin embargo, si hay sintomatología lo más frecuente es dolor abdominal, náuseas, vómitos, sangrado y disfagia ${ }^{13}$. Según lo reportado en la literatura, encontramos que varía del $1 \%$ al $16 \%$ aproximadamente y existen numerosos factores asociados a su aparición, como el uso crónico de AINE, el abuso de psicoactivos, el consumo de tabaco, la anticoagulación crónica y la infección por $H$. pylori, entre otros ${ }^{14}$.

En cuanto a los factores de riesgo, Steinman, et al. ${ }^{12}$ realizaron un estudio en el que los dividieron en quirúrgicos y no quirúrgicos. Los factores de riesgo quirúrgicos son isquemia en la mucosa, tensión de la anastomosis, gran pouch gástrico con mayor producción celular de gastrina e interrupción en la línea de grapas $^{12}$. Los factores de riesgo no quirúrgicos son uso de AINE, presencia de H. pylori, tabaquismo, diabetes mellitus y gran pouch gástrico expuesto a ambiente ácido en la gastroyeyunostomía ${ }^{12}$.

Los pacientes van a cursar en general con náuseas, pirosis y disfagia; síntomas que van a relacionarse inicialmente con una excesiva ingesta de alimentos en el posoperatorio. Tanto en la prevención como el manejo debe incluirse el tratamiento con un inhibidor de la bomba de protones, ya que esto se ha asociado a una disminución en la incidencia de úlceras, así como la erradicación de los factores de riesgo involucrados y el control endoscópico ${ }^{4,11,15}$.

Las complicaciones de la úlcera marginal pueden ser variadas, incluyendo sangrado, estenosis, malignización o perforación; esta última ocurre en menos del $1 \%$ de los casos, pero puede llegar a ser fatal, lo cual nos obliga a sospecharla en los pacientes con antecedente de bypass gástrico y abdomen agudo, con el fin de poder brindar tratamiento oportuno y eficaz por medio de la cirugía de emergencia con parche de omento, con la cual generalmente se obtienen buenos resultados ${ }^{16,17}$.

La rotura de una úlcera marginal es una complicación con una baja incidencia, pero con un alto potencial de mortalidad ${ }^{6,7,9,15}$. Es una urgencia que debe ser atendida inmediatamente y con una incidencia aproximada del $0.9-1 \%$. La reparación mediante técnica laparoscópica y reforzamiento con parche omental (parche de Graham modificado) es el procedimiento de elección en estos casos en los que se debe manejar la perforación. Ha demostrado que es una técnica segura y además ofrece los beneficios de la cirugía por laparoscopia, como menor estancia hospitalaria, menos dolor posoperatorio y un tiempo de 
recuperación más corto, como sucedió en este caso. En los pacientes con sangrado masivo se utiliza también la sutura endoscópica, que se considera una técnica segura y efectiva para el tratamiento en estos casos de urgencia como alternativa a la cirugía $a^{4,6,7,11}$.

Wendling, et al. ${ }^{17}$ realizaron un estudio para comparar la reparación con parche omental y la cirugía de revisión de anastomosis en la úlcera perforada, y concluyeron que el parche omental es más seguro y efectivo, ya que requiere menor estancia hospitalaria y produce menor sangrado. La tasa de recurrencia tras la reparación con revisión anastomótica de una úlcera marginal es relativamente alta: $26.09 \%$ para la reparación y $29.69 \%$ para la revisión. Por otra parte, Marambio, et al. ${ }^{18}$, en un estudio realizado en la Universidad de Chile durante un periodo de 10 años, reportaron que solo el $0.57 \%$ de los pacientes llevados a bypass gástrico desarrollaron úlcera marginal, de los cuales la mayoría fueron mujeres, con un promedio de edad de 39 años y que desarrollaron esta complicación en promedio a los 27 meses del posoperatorio. Estos autores concluyen que los pacientes se benefician del manejo laparoscópico porque presenta baja mortalidad, y que el desarrollo de esta complicación está altamente relacionado con el hábito tabáquico. Por lo tanto, la vigilancia cercana y las medidas antiúlcera a largo plazo benefician a los pacientes, en especial a los de alto riesgo de desarrollar úlceras marginales tras un bypass gástrico ${ }^{6}$.

\section{Conclusión}

Las úlceras marginales gástricas en pacientes sometidos a un bypass gástrico son una patología que se debe sospechar en aquellos pacientes con factores de riesgo. Ante su sospecha, requiere un adecuado seguimiento para evitar su rotura. En caso de requerir manejo quirúrgico de urgencia por perforación, la técnica más aceptada y segura es la reparación laparoscópica con reforzamiento de parche omental.

\section{Agradecimientos}

Nuestros más sinceros agradecimientos a nuestra alma mater, Universidad El Bosque, y especialmente a todos los integrantes del Departamento de Cirugía General, en particular a sus docentes, quienes han velado y perseverado por impartir sus conocimientos con humildad en cada uno de sus estudiantes. También agradecemos a todas aquellas personas que de una u otra forma aportaron algo para la elaboración de este reporte. Tenemos la firme convicción de ser cada día mejores manteniendo en alto el pilar de que en nuestra profesión el aprendizaje es a diario y la pasión por lo que hacemos es la constante.

\section{Conflicto de intereses}

Los autores declaran no tener ningún conflicto de intereses y no tener relación comercial, profesional ni financiera con ningún patrocinador. Este caso clínico únicamente tiene fines académicos.

\section{Responsabilidades éticas}

Protección de personas y animales. Los autores declaran que para esta investigación no se han realizado experimentos en seres humanos ni en animales.

Confidencialidad de los datos. Los autores declaran que han seguido los protocolos de su centro de trabajo sobre la publicación de datos de pacientes.

Derecho a la privacidad y consentimiento informado. Los autores han obtenido el consentimiento informado de los pacientes y/o sujetos referidos en el artículo. Este documento obra en poder del autor de correspondencia.

\section{Bibliografía}

1. Marcotte E, Sweigert PJ, Bakhos F, Chand B. Treatment of marginal ulcers after gastric bypass. ResearchGate, 2011. Chapter 11. p. 119-28. Disponible en:

2. Coblijn UK, Goucham AB, Lagarde SM, Kuiken SD, van Wagensveld BA. Development of ulcer disease after Roux-en-Y gastric bypass, incidence, risk factors, and patient presentation: a systematic review. Obes Surg. 2014 Feb;24(2):299-309.

3. Csendes A, Torres J, Burgos AM. Late marginal ulcers after gastric bypass for morbid obesity. Clinical and endoscopic findings and response to treatment. Obes Surg. 2011;21(9):1319-22.

4. Wheeler AA, de la Torre RA, Fearing NM. Laparoscopic repair of perforated marginal ulcer following Roux-en-Y gastric bypass: a case series. J Laparoendosc Adv Surg Tech A. 2011;21(1):57-60.

5. Pohl D, Schmutz G, Plitzko G, Kröll D, Nett P, Borbély Y. Perforated duodenal ulcers after roux-Y gastric bypass. The American Journal of Emergency Medicine, 2011.

6. Altieri M, Pryor A, Yang J, Yin D, Docimo S, Bates A. The natural history of perforated marginal ulcers after gastric bypass surgery. Surgical Endoscopy, 2018.

7. Wang E, Blackham R, Tan J, Hamdorf J. Giant perforated marginal ulcer after laparoscopic Roux-en-Y gastric bypass. BMJ Case Rep. 2017: bcr2016218829. doi: 10.1136/bcr-2016-218829.

8. Pang AJ, Hagen J. Laparoscopic approach to a bleeding marginal ulcer fistulized to the gastric remnant in a patient post Roux-en-Y gastric bypass. Surgery for Obesity and Related Diseases. 2017.

9. Nimeri A, Maasher A, Al Shaban T. Efferent limb obstruction and unexpected perforated marginal ulcer in a pregnant patient after one anastomosis gastric bypass/mini gastric bypass. Surgery for Obesity and Related Diseases. 2017.

10. Godina M, Nagliati C, Menegon P, Caruso V. Emergency laparoscopic conversion from mini/one anastomosis gastric bypass to modified Rouxen-Y-gastric bypass due to acute bleeding from a recurrent marginal ulcer. Updates Surg. 2017;69(3):421-4. 
11. Barola S, Magnuson T, Schweitzer M, Chen YI, Ngamruengphong S, Khashab MA, et al. Endoscopic Suturing for Massively Bleeding Marginal Ulcer 10 days Post Roux-en-Y Gastric Bypass. Obes Surg. 2017;27(5):1394-6.

12. Steinemann DC, Bueter M, Schiesser M, Amygdalos I, Clavien PA Nocito A. Management of anastomotic ulcers after Roux-en-Y gastric by-pass: results of an international survey. Obes Surg. 2014;24 (5):741-6.

13. Moon RC, Teixeira AF, Goldbach M, Jawad MA. Management and treatment outcomes of marginal ulcers after Roux-en-Y gastric bypass at a single high volume bariatric center. Surg Obes Relat Dis. 2014;10.

14. El-Hayek K, Timratana P, Shimizu H, Chand B. Marginal ulcer after Roux-en-Y gastric bypass: what have we really learned? Surg Endosc. 2012;26(10):2789-96.
15. Chang $P$, Huang $C$, Tai $C$, Huang I, Hsin M, Hung C. Revision using totally hand-sewn gastrojejunostomy and truncal vagotomy for refractory marginal ulcer after laparoscopic Roux-en-Y gastric bypass: a case series. Surgery for Obesity and Related Diseases. 2017;13.

16. Kalaiselvan R, Exarchos G, Hamza N, Ammori BJ. Incidence of perforated gastrojejunal anastomotic ulcers after laparoscopic gastric bypass for morbid obesity and role of laparoscopy in their management. Surg Obes Relat Dis. 2012;8.

17. Wendling M, Linn J, Keplinger K, Mikami D, Perry K, Melvin WS. Omental patch repair effectively treats perforated marginal ulcer following Roux-en-Y gastric bypass. Surg Endosc. 2013;27.

18. Marambio A, Gabrielli M, De la Llera J, Crovari, F, Pérez, G. Úlcera marginal perforada post bypass gástrico laparoscópico. Revista Chilena de Cirugía. 2015;67. 